\title{
El Estado: acercamiento a la sociedad actual
}

\section{INTRODUCCIÓN}

Proyectos, métodos, orientaciones, «imaginación al poder», «dinamización» de la economía, de la sociedad, ... son algunos de los términos más utilizados por los medios de comunicación «social», por los políticos, por los teóricos del pensamiento. Es el lenguaje vigente en el medio en que nos movemos, reflejo y estímulo de una sociedad que nos va conformando. La dinámica social en nuestros días se define por el «proyectismo»: ;programación o muerte!. O, lo que es lo mismo, actividad continua, desarrollo ilimitado, o muerte. Naturalmente nos referimos a la defunción del actual sistema socioeconómico, basado en una elevación «ad infinitum» del nivel de vida, de las necesidades artificiales.

La institución axial de nuestra sociedad es la económica. La preocupación fundamental es apuntalarla, que no «se nos caiga», que no cree paro. $Y$ desde el apuntalamiento, la expansión: el crecimiento «ad infinitum» se constituye en cuestión de supervivencia, en sentido social: a él nos ordenamos la «masa anónima» que posibilitamos la misma sociedad. La planificación económica cataliza los mayores esfuerzos, en torno a ella se organizan las actividades y se impele a la acción. Es necesario que la gente compre, que las necesidades aumenten, que el nivel de vida (nivel desde donde se vive) se artificialice aún más... La teleología consumista.

¡Invierte!, ¡consume!, ¡ayuda a tu sociedad!... El fenómeno económico ejemplifica este proceso de «independencia» de la sociedad respecto del individuo, de la persona. El Estado, que es una parte del cuerpo político especializada en los intereses del todo ${ }^{1}$, deja de estar al servicio del hombre: este haz de

1. Maritain, J., El hombre y el estado, Buenos Aires, Kraft 1952, pp. 25-26. 
instituciones combinadas, ordenadas al bienestar público, acaba por situar como norte de su actividad su propio mantenimiento.

Programar hace referencia a una actividad metodológica: consiste en la formulación de líneas de actuación para conseguir un fin. ¿Cuál es el fin al que se encaminan los esfuerzos de nuestra sociedad? El sistema identifica, y hace que identifiquemos, el «caos» con su desmantelamiento: paro, miseria, inestabilidad. Pero,... ¿y el hombre? Estamos ordenados al sistema... el sistema es nuestra vida. Sus necesidades son las nuestras. Surge el gran interrogante: ¿no será que, de ser la respuesta a nuestras necesidades, la sociedad/sistema ha pasado a dictarlas?

Asistimos a la independencia del Estado, de lo impersonal, del «sistema», de la realidad-base que le sustenta: la sociedad. Frente a la seguridad ficticia, a la felicidad pasiva del tener que otorga el Estado/sistema, frente a su proclama, un dato empírico que la refuta: el resquebrajamiento de la persona, elemento fundante de la sociedad; diversos intelectuales de nuestros días predican la «muerte del hombre», más aún, a menudo se vive esta «muerte». El hombre de nuestro tiempo es el ser más alienado de la historia: satisface una serie de necesidades externas, impuestas por el Estado/sistema, ajenas a su propia realidad. Consecuencia lógica es la pérdida de sentido vital, la desorientación, el desaliento. El doblegamiento del ser humano, su sometimiento, se facilita, y la espiral sigue.

Es necesario, pues, plantearse en serio el problema clave de nuestro tiempo: las necesidades del hombre, su realización personal y social, la finalidad de la sociedad y del Estado. Tenemos que ubicarnos en una correcta perspectiva sociológica, a fin de situar en el lugar correspondiente al hombre, a la sociedad, al Estado. Hay que clarificar conceptos, y a partir de ahí analizar la sociedad actual, proponiendo caminos adecuados de superación de los defectos que observemos.

El tema es tan amplio como urgente. Por obvias limitaciones, intentaré en el presente trabajo ubicar la realidad Estado sociológicamente, y poner las bases para estudios posteriores.

\section{ACERCAMIENTO HISTÓRICO-FILOSÓFICO: EL ESTABLECIMIENTO DEL ESTADO}

Como paso previo a la correcta comprensión sociológica de la realidad Estado, y de su razón de ser (satisfacer las necesidades del hombre), haremos un breve recorrido por las diversas nociones históricas del mismo:

\section{- Antigüedad}

El problema del Estado es un caso particular del problema más general de 
la justicia: Platón, Aristóteles, etc., hablan del Estado como la mejor organización de la sociedad, como aquella forma o articulación de los individuos y de las clases que permite realizar en la medida de lo posible la idea de la justicia, dando a cada uno lo que de derecho le pertenece.

\section{-Edad Media}

Se disputa acerca de la supremacía del Estado sobre la Iglesia, o viceversa. Entendían por Estado una comunidad temporal e histórica, y concluían la inferioridad de éste respecto de la Iglesia.

\section{-Renacimiento}

El Estado es desvinculado de su fundamento divino, (primado del Estado, separación de la Iglesia, origen y transmisión humana del poder -Maquiavelo--), y decididamente insertado en la temporalidad y en la historia. Las diversas teorías utópicas intentan encontrar una organización de tal índole que sea posible en ella la paz y la justicia.

-Siglos XVII-XVIII .

Surgen dos corrientes principales:

I. Teoría del Estado como pacto: Se origina para evitar el aniquilamiento final en lá guerra (Hobbes), como renuncia al egoísmo producido por el Estado natural incivilizado y consiguiente: sometimiento a la voluntad general (Rousseau).

II. Teoría del Estado como comunidad de hombres libres: El Estado es aquella organización de la sociedad que garantiza la libertad, cuyo fin es la libertad. Los hombres son más libres porque viven en el Estado «según el decreto común» (Spinoza).

\section{-Ilustración: despotismo ilustrado}

Para ellos es la organización que puede conducir a los hombres por el camino de la Razón frente a las tinieblas del pasado.

\section{- Kant}

El Estado debe estar constituido de tal modo que la ley corresponda a una organización establecida por pacto y contrato. La libertad (respecto de la libertad moral de cada uno a la libertad moral del conjunto hecha posible por la ley) es el fin del Estado. Los componentes del Estado son hombres, fines en sí que deben someterse al fin en sí de su moralidad.

\section{-Filosofía romántica alemana}

Identifican Nación y Estado. Éste encarna todas las funciones del pueblo, 
es el lugar donde el espíritu objetivo se realiza plenamente (Hegel). El que rige el Estado debe ser el representante del «espíritu nacional», el que cumple los fines objetivos planteados por este espíritu.

-Siglo $X I X$

El individualismo concibe al Estado como el equilibrio de la tensión entre las voluntades particulares.

El colectivismo lo concibe como el equilibrio resultante de la supresión de esas voluntades, cuya presencia y actuación se suponen nocivas para el Estado.

La supresión de la tensión entre clases por una dictadura aparece en:

1) Marxismo: dictadura proletaria. El Estado representa el dominio de una clase.

2) Estados totalitarios: toda actividad queda integrada en el cuerpo del Estado, identificado con un partido que pretende representar a la nación, pueblo, etc. Queda excluido todo lo que no se halle al servicio del Estado: tienden a divinizarlo.

\section{UBICACIÓN SOCIOLÓGICA DE LA REALIDAD ESTADO}

\section{El hecho social: De las necesidades ${ }^{2}$.}

En último término la unidad física irreductible de la sociedad es la persona social. Desde el punto de vista de las personas que la constituyen, una sociedad es un gran número de seres humanos que obran conjuntamente para satisfacer sus necesidades sociales y que comparten una cultura común. Se puede decir en general que el grupo está constituido por personas, y que la sociedad está constituida por grupos. En un primer acercamiento podríamos definir a la sociedad como la estructura formada por los grupos principales interconectados entre sí, considerados como una unidad y participando todos de una cultúra común.

Englobando las características principales de la sociedad (unidad demográfica, zona geográfica común, unidad constituida por grandes grupos que se diferencian por su función social, unidad que funciona en todas partes, unidad social separada), podemos formular la siguiente definición: Una sociedad es una colectividad organizada de personas que viven juntas en un territorio

2. Fichter, J.H., Sociología, trad. A.E. Lator Ros, Herder, Barcelona 1982, pp. 153-157. 
común, cooperan en grupo para satisfacer sus necesidades sociales básicas, adoptan una cultura común y funcionan como una unidad social distinta.

Evidentemente, la sociedad existe para las personas. Desempeña ciertas funciones generales a través de las funciones más específicamente determinadas que son propias de los grupos principales. Estas funciones generales son las siguientes: hacer posible las mutuas relaciones humanas, la intercomunicación, proporcionar pautas comunes de comportamiento y un sistema de estratificación de status y clases.

Las funciones más específicas de la sociedad giran en torno a las soluciones que dan los grupos a las primarias y básicas necesidades sociales de las personas: posibilita la renovación de sus miembros, la socialización, la producción de los bienes y servicios materiales y físicos necesarios, el orden y la seguridad externa, la atención de las necesidades religiosas y espirituales, el descanso. La sociedad se compone de personas por medio de las cuales «ayuda» a otras personas a nacer, a criarse, a educarse. Sin la sociedad la persona no encontraría el adecuado apoyo material, la protección política, y las múltiples oportunidades religiosas y recreativas.

Así pues, la sociedad es la respuesta colectiva a las necesidades comunes.

\section{La articulación social:}

\subsection{Aspecto estático: estar.}

El primer hecho social consiste en las diversas maneras de estar juntos: la sociedad aparece estructurada. La estructura hace referencia a la organización de sus partes o unidades. Responde a la pregunta: ¿cómo está dispuesta la sociedad? Vamos a exponer sintéticamente esas diversas maneras de estar juntos:

-Categorías sociales:

Pluralidad de personas que se consideran formando una unidad por el hecho de ser semejantes en uno o varios aspectos.

-Conglomerados:

Conjunto de personas que están juntas físicamente, consideradas en su totalidad, pero sin comunicación recíproca.

-Grupos:

«El grupo es una colectividad identificable, estructurada, continuada, de personas sociales que desempeñan funciones recíprocas conforme a determinadas normas, interéses y valores sociales para la prosecución de objetivos comunes" ${ }^{3}$.

3. FICHTER, J.H., o.c., p. 109. 
-La sociedad:

La sociedad es un conjunto de grupos interconexionados entre sí, que tienen una cultura y realizan funciones principales para la persona: la persona humana junto con los demás formando grupos es quien participa de la cultura $\mathrm{y}$ de las funciones principales.

\subsection{Aspecto dinámico: satisfacer}

La función de una sociedad responde a la pregunta: ¿qué hace la sociedad? La sociología debe estudiar lo que «son» las personas sociales, su articulación en grupos, y debe estudiar también lo que «hacen», cómo responden a sus necesidades básicas mediante los grupos y sociedad que forman. Partiendo de la unidad mínima, la pauta de cultura, haremos un breve análisis de los diversos modos de hacer:

-Pautas de conducta:

Comportamientos estandarizados que realizan los individuos, y que juzgan deben realizarse así: modos de comportamiento que sirven de modelo de lo que ha de ser aceptado o no; es pauta en cuanto sirve de modelo, y es norma en cuanto es lo deseable y exigible en la sociedad. Están agrupadas en torno a los roles.

-Roles:

Conjunto de pautas de comportamiento relacionadas entre sí que se agrupan en torno a una función social. El rol social se determina y se especifica por la necesidad social a que responde y por el grupo sociäl en el que se desempeña. El sistema total de roles por los que el individuo «trata» con la sociedad constituye la personalidad social.

-Procesos sociales de interacción:

Modos de realizar nuestros roles en interacción con los demás: formas básicas tipificables de interacción social que se cruzan a través de los numerosos roles que desempeñan los individuos.

-Instituciones:

«Una institución es una estructura relativamente permanente de pautas, roles y relaciones que las personas realizan según unas determinadas formas sancionadas y unificadas, con objeto de satisfacer necesidades sociales básicas) ${ }^{4}$. Toda institución tiene un fin (las necesidades básicas), es relativamente permanente (porque lo son las necesidades que solventa), está estructurada y dice relación con otras para formar un todo orgánico que tiende a dar cohe-

4. FICHTER, J.H., o.c., p. 248. 
sión, es una unidad netamente diferenciable de cualquier otra institución (aunque no funciona de modo independiente), está cargada de un fuerte valor en relación a las necesidades que cubre.

-Cultura:

Conjunto trabado de pautas sociales (internas y externas), roles, procesos de interacción e instituciones sociales que están en vigor en una sociedad, y que permiten la comunicación y las relaciones sociales entre los miembros de dicha sociedad.

\section{El Estado: ubicación sociológica}

Tras este acelerado repaso de la sociedad, en su doble aspecto estático y dinámico; ¿dónde situar el Estado?

Volviendo a la definición que da Maritain del Estado en su obra «El hombre y el Estado», citada en la introducción, podemos concebir el Estado como ese haz de instituciones combinadas ordenadas al bienestar público. Es, pues, la «super-institución», la institución social por excelencia, aquella que regula la correcta orientación de las demás instituciones. Ahora bien, ¿en qué se diferencia de la Cultura? La Cultura es el modo peculiar de responder de cada sociedad a sus necesidades básicas. El Estado es un regulador de ese modo, un delimitador. La Cultura es el sistema total («configuración total de las instituciones que comparten en común las personas de una sociedad») ${ }^{5}$. El Estado es el medio por excelencia que se otorga a sí misma la sociedad para asegurar la correcta satisfacción de las necesidades básicas. No puede identificarse el Estado con la sociedad, ni con la cultura total de una sociedad. Rechazamos, pues, la concepción que sobre el Estado defendía la filosofía romántica alemana: da lugar a ciertos «despropósitos» (nazismo, etc.) por todos conocidos..,

«El cuerpo político o sociedad política es el todo. El Estado es una parte - la más sobresaliente- de ese todo (...). Debería resultar evidente que el cuerpo político difiere del Estado. Éste es sólo aquella parte del cuerpo político especialmente interesada en el mantenimiento de la ley, el fomento del bienestar común y el orden público, así como la administración de los asuntos públicos. El Estado es una parte que se especializa en los intereses del todo (...), organismo facultado para utilizar el poder y la coerción, integrado por expertos o especialistas en ordenamiento y bienestar públicos, un instrumento al servicio del hombre, (...) parte o instrumento del cuerpo político, subordinada a él y dotada de la máxima autoridad, no por derecho propio ni para su

5. Idem, o.c., p. 272. 
beneficio, sino únicamente en virtud de y para el cumplimiento de las exigencias del bien común» ${ }^{6}$.

El Estado, la super-institución, responde a la pretensión social de conseguir la universalidad de la justicia y el bienestar; en principio sólo actúa en aquellas áreas en las que es necesaria su presencia para la consecución del bien común. Las características de las instituciones le corresponden, pero amplificadas en un enorme grado: el proceso de «institucionalización» es mucho mayor, así como el fenómeno de difuminación de la responsabilidad social: la excesiva complejidad de la sociedad actual; junto con la super-especialización de las actividades y del trabajo, produce en las personas una «permanente» situación de confusión respecto al funcionamiento social, y respecto a la toma de opciones sociales, de nuevos proyectos de transformación seria de la sociedad. Se produce una disminución de la conciencia personal, nadie se siente responsable, y se delega en el Estado... De ahí su formidable poder, no sólo de regulación, sino de «conformación». La problemática se presenta con tales proporciones y dificultades, tan lejos de las posibilidades de cada cual, que la indiferencia y el «sálvese quien pueda» parecen ser las únicas posturas razonables, o al menos las únicas practicables. El Estado es el que ha de solucionar los problemas. La persona remite a la «super-institución», y ésta acaba por convertirse en isujeto social!, haciendo de aquélla un mero peón de sus resoluciones. Más adelante volveremos sobre esto, pero dejando ya claro que este boceto de hiperestado es una desviación de la genuina realidad Estado, en parte peligro y en parte realidad en nuestra sociedad.

\section{Intento de comprensión de nuestra sociedad y Estado actuales}

En las líneas anteriores ya hemos dado un primer paso, al describir el fenómeno de difuminación social que se da en nuestra sociedad super-tecnificada, y su repercusión en la potenciación del Estado.

La sociedad es dinámica. A la vez que va complejizándose, va creando mecanismos que regulen ese crecimiento; se va dando reglas conforme a la propia necesidad que experimenta de ordenar sus fuerzas hacia la consecución del bien común. De su propia conciencia (de la conciencia de las personas sociales y grupos que la componen) brotan ciertas evidencias, como la igualdad de todos sus componentes ante la ley, etc., tal y como conciben las personas sociales y los grupos de cada sociedad esa igualdad, esa ley, y todo lo demás. Es «consciente» de lo bueno y de lo malo (de acuerdo con su mentalidad), y en

6. Maritain, J., o.c., pp. 22-27. 
conformidad legisla. Lo bueno y lo malo hacen referencia, en último lugar, a la satisfacción o no de las necesidades básicas, de las que tiene pleno conocimiento.

En las sociedades primitivas, por ejemplo, existía una fuerte conciencia del sentimiento de grupo, así como de los deberes y necesidades del mismo y de sus miembros. La comprensión de la estructura (mínima) social, de las necesidades y de los medios para solventarlas era evidente a los ojos de las personas sociales y de los grupos (en la medida en que pueda hablarse de ellos) que la componían.

A medida que la sociedad va desarrollándose se producen dos fenómenos: diversificación de las necesidades, y consecuente especialización del trabajo, junto con una complejización cada vez mayor de las estructuras sociales. Las personas sociales van perdiendo esa visión global de la sociedad, disminuyendo su capacidad de comprensión, de gobierno y de proponer alternativas. La sociedad se independiza, poco a poco, de sus «componentes».

Surgen los límites territoriales, las legislaciones, (cada vez más complicadas, extensas y pormenorizadas), etc. Para evitar que se dañe el bien común, o que no se potencie, para velar por el cumplimiento de la universalidad de la justicia (tal como se concibe en cada sociedad) la sociedad desarrolla su propio mecanismo de autoprotección: surge el Estado. En su origen es un instrumento pasivo, es el conjunto de todos esos medios de que se ha ido dotando la sociedad. Su objetivo es la satisfacción de las necesidades básicas, o mejor dicho, la vigilancia de la correcta satisfacción de las mismas.

Con la expansión demográfica, económica, cultural, se va tornando mayor el distanciamiento entre el bien social objetivo y la conciencia que tienen de él los grupos sociales. Así aparecen distintas teorías y movimientos cuya finalidad no es la plena realización de los seres humanos como seres sociales: nos referimos, por ejemplo, al individualismo-egoísmo de Mandeville, a la despersonalización marxista o fascista, etc. Estas concepciones parten de una incorrecta comprensión de la realidad social circundante, de las necesidades básicas del ser humano, y han dado pie a modelos sociales concretos deshumanizadores, desplenificadores, alienadores. El oscurecimiento progresivo del bien social en la mente de los componentes de la sociedad se tipifica en la convicción de algunos ilustrados de que el motor del progreso social es el egoísmo individual, convirtiendo, con frase de Carlos Díaz, los vicios privados en virtudes públicas...

En este crecimiento social crece, también, el Estado. Frente a las desviaciones, prácticas o teóricas, el Estado se expande como única solución: amplifica sus medios de intervención en la sociedad, y comienza a delimitar, cada vez con más detalle, las acciones sociales. Defiende el orden establecido, o lo 
que se establece, buscando en principio la universalidad del bien común y de la justicia.

La complejización de la sociedad concluye en una independencia de ésta sobre el individuo, que se ve superado, sin capacidad de acción. La pérdida de la conciencia social en las personas sociales y en los grupos que componen la sociedad provoca un debilitamiento social, una ausencia de proyecto y categorías comunes. Tras esta pérdida, los grupos sociales y las personas sólo aspiran al automantenimiento, a la realización propia, a dejar las cosas como están, al menos en lo fundamental: la prioridad no será ya analizar el sistema socio-económico, y si se da el caso cambiarlo. La prioridad es mantenerlo y potenciarlo; el lema sería integrarse en la sociedad de consumo: «Realización» individual, comodidad, situarse cada uno donde mejor pueda, indiferencia...

El complejo socio-económico, independizado, aspira a la autopermanencia. La masa social se ordena a este objetivo, presa de la propia dinámica del sistema, y acaba por identificar esa permanencia con su propia realización. Los medios de comunicación ya se encargan de «demostrarnos» que el paraíso es el consumo, que el hombre «auténtico» viene de la mano de la colonia Yacaré... Se busca la plena docilidad del telespectador.

El Estado, creación máxima de la sociedad, va adquiriendo una autonomía cada vez mayor. Las responsabilidades sociales se remiten a él: educación, justicia, orden público... El Estado se potencia. De ser un elemento pasivo, a la espera del desvío social, ordenador de las fuerzas sociales cuando la satisfacción del bien común así lo exija, el Estado pasa a transformarse en un elemento activo, tremendamente condicionador de la sociedad: da ya las respuestas, señala horizontes, cuando en un principio sólo encauzaba la respuesta errónea hacia la correcta, ya establecida por la sociedad. El Estado es quien sanciona, más aún, quien moraliza, quien decide lo bueno y lo malo: con un ejemplo de Carlos Díaz, el Estado exhorta a ser fieles a Hacienda (en definitiva a él mismo) mientras casi estimula el que le pongamos los cuernos a Purita... La doble contabilidad moral. Nos responsabiliza respecto a él, y nos libera de la responsabilidad social auténtica: pagamos los impuestos religiosamente, mientras que, en una calle céntrica de París, violan a nuestro lado a una joven... isin suscitar la mínima reacción de defensa, de ayuda, en los transeúntes! Lo único que se les ocurre es lamentar la «suerte» de la pobre chica, y quejarse por la ausencia de la policía, en definitiva, por la ausencia del Estado, que es quien debe «defendernos». Se ha conseguido la pasividad absoluta del ciudadano. ¿Podría suceder algo así en un pueblo pequeño, o en una sociedad tribal?

El proyecto social originario, orientado a la satisfacción plena de las necesidades básicas, se ha desvanecido aquí. La institución económica, la fami- 
liar, la recreativa, la educativa, la política, el Estado, se han independizado de su finalidad fundante, de su razón de ser, el hombre como ser social que intenta realizarse. Ellas mismas se convierten en sus propias razones de ser. Naturalmente, esto produce un elevado grado de insatisfacción entre los componentes de la sociedad, una alienación continua. Quizás sea este proceso una de las causas que pueden explicar el consumo de droga, la infelicidad de los bien situados, así como la «huida» hacia los «grupos primarios». Y digo huida porque tiene uno la impresión de que las personas buscan en esos grupos una vía de escape, que les permita una mínima realización, pero sin plantearse un análisis científico de la realidad social que viven, que les empuja a esos grupos, y por lo tanto, sin proponer un nuevo planteamiento de transformación de la sociedad actual: «No se puede hacer nada, lo que hay que hacer es intentar vivir nosotros lo más plenamente posible (plenamente entre comillas), montárnoslo lo mejor que podamos»... Un individualismo cómodo. No se afronta la sociedad tal como es, se agacha la cabeza. De este modo el sistema siempre nos gana la partida.

Nuestra sociedad super-tecnificada ha desarrollado un Estado con amplios poderes de actuación. Ahora bien, el Estado no es un ser personal, no se le puede concebir como algo biológico con vida propia. Es un instrumento, un enorme instrumento creado por la sociedad, y que por lo tanto remite a ella: no es posible concebirlo en términos substancialistas. El Estado, por la propia dinámica de toda institución, tiende a ampliarse, a abarcar un campo de acción cada vez mayor, pero no posee ideología propia, pensamiento propio.

Establecido esto, señalamos brevemente dos peligros que acechan nuestra sociedad:

-El Estado es un mecanismo de la sociedad. A nuestra sociedad independizada de las personas sociales, y de su deber de satisfacer las necesidades básicas, le corresponde un Estado que, como ella, centra su atención en su propio mantenimiento, y en el mantenimiento del actual sistema socio-económico. El primer peligro sería la teleología estatal. Un ejemplo de la fuerza devoradora del sistema, y del Estado, así como de la carencia de un auténtico proyecto social, sería la «peripecia» del actual gobierno socialista: llegaron al poder sin un proyecto social serio, de la mano de slogans, y una vez en él han dejado de lado las proclamas electoralistas para conformarse al sistema socio-económico. Su objetivo no es ya «transformar», sino «potenciar» el sistema y el Estado; se han vuelto prácticos... Les ha vencido el Poder. Son los nuevos paladines de la sociedad de consumo. La consistencia de su oferta se mide por su fracaso, por su renuncia voluntaria. Lo cual es todo un símbolo de la carencia de un verdadero proyecto de una nueva sociedad más acorde con el «sistema hombre», utilizando la terminología de Fromm, así como de la capacidad 
conformadora del sistema, que se esconde tras el cebo de un bienestar material caduco y corto de miras, meta añorada, podría decirse que «idealizada», por nuestra sociedad del paro.

-El segundo peligro proviene de una falsa concepción que puede extenderse entre nuestros políticos: la de su propia omnipotencia respecto de la sociedad. Nos referimos al problema de la representatividad social: el Estado responde a la necesidad social de asegurar la satisfacción de las necesidades básicas. En su origen estos medios expresaban lo que quería la sociedad (por ejemplo, la igualdad de todos los ciudadanos ante la ley). Nuestro Estado actual dispone de medios enormes, más aún, posee la capacidad de adoctrinarnos. Este Estado, que responde a la dinámica interna de la sociedad, es mucho más amplio que lo que comúnmente entendemos por política; las funciones de ésta no abarcan la totalidad de las funciones del Estado. No puede identificarse el Estado y su legitimidad, dentro de sus justos límites, con la corriente política que triunfa en las elecciones, ni puede arrogarse ésta la potestad de manejar impunemente a aquél: es un grave error considerar al Estado como un trofeo electoral, que una vez obtenido no tiene otra función que la realización de los deseos de la ideología dominante. Al defender esta visión de la sociedad y del papel de la política nos situamos a un paso del absolutismo, de la dictadura, y comenzamos a caminar hacia el adoctrinamiento, hacia la muerte de la vida social. Las ideologías políticas concretas son movimientos sociales «temporales», con unas funciones determinadas, frente al Estado, que precisamente por ser algo mucho más amplio, el complejo conjunto de mecanismos con los que la sociedad regula la satisfacción de sus propias necesidades, puede recibir el calificativo de «intemporal»: las corrientes políticas pasan, la sociedad y el Estado permanecen. Allí están las elecciones generales cada cuatro años para recordárnoslo. Hay que respetar mucho más la sociedad.

\section{CONCLUSIÓN}

\section{El Estado}

El Estado es el regulador se ese todo orgánico que tiende a formar el conjunto de las instituciones. Participa de las características de éstas, pero en él sufren una gran potenciación. El Estado es un instrumento que la sociedad se otorga a sí misma, que surge de su propia dinámica de crecimiento para asegurar el bien común. Refleja la esencia de toda sociedad: la satisfacción de las necesidades básicas de todos sus componentes; pretensión intrínseca del Estado es la universalidad del bien común. Por esto es algo mucho más amplio que lo político, y a lo que lo político tiene acceso, acceso que debiera estar limita- 
do por la propia conciencia de la realidad social: el aparato judicial, los controles de todo tipo que han brotado de la sociedad para su correcta orientación, etc., constituyen una auténtica «representación social», quizás la más compleja, de lo que es y quiere ser la sociedad. De aquí brota la legitimidad profunda del Estado, siempre que se mantenga dentro de sus justos límites. De aquí que sea mucho más amplio que las corrientes políticas concretas, porque surge de toda la sociedad, reflejando su finalidad fundante: la plena realización del hombre. Ninguna corriente política puede arrogarse este grado de representatividad, y por lo tanto no es omnipotente respecto del Estado y de la sociedad. Sería interesante un estudio que delimitara las funciones de ambas realidades sociales, sus ámbitos y límites, estudio que estableciera el primado de la sociedad: vistas así las cosas, diez millones de votos no son un cheque en blanco. Eliminar la división de poderes, por ejemplo, aparece, tras una adecuada ubicación sociológica, como algo antinatural, una prerrogativa vanidosa de quien no conoce la sociedad, ni sus necesidades, ni sus mecanismos, ni la función de la política. Constituye el resultado de una extensión indebida de la representatividad social que suponen las elecciones: no se puede reducir toda la sociedad a una ideología concreta.

En principio el Estado es un instrumento pasivo de la sociedad, que no engloba todas las instituciones, sino que sólo actúa en determinadas áreas para asegurar el bien común: ante un desvío de las fuerzas sociales, conduce a éstas a lo previamente establecido por la sociedad: a la satisfacción de las necesidades básicas. Responde, pues, al estímulo social. Nuestra macro-sociedad ha desarrollado un Estado acorde con sus necesidades de regulación: un gigantesco Estado, caracterizado por la gran cantidad de atribuciones que ha delegado en él nuestra sociedad. La debilidad social potencia el crecimiento del Estado. El gran peligro de nuestra sociedad es la independencia que ésta ha conseguido sobre el ser humano concreto y sus necesidades, independencia que también caracteriza a la mayor creación social: el Estado. Quizás aquí halle una explicación la creciente insolidaridad del individuo para con la sociedad/sistema, y para con el Estado; éste, de ser algo «nuestro», pasa a ser un jenemigo! a burlar...

Por último, conviene resaltar el papel conformador que ha ido adquiriendo el aparato estatal, abandonando su primitivo carácter pasivo: la pérdida de la orientación social, de la conciencia de responsabilidad cívica, deja a la persona a merced del sistema socio-económico y del Estado, que se convierten en sujetos sociales activos, orientados a su propio automantenimiento y potenciación, sujetos que adoctrinan a los hombres, convirtiéndolos en objetos al servicio de sus propias finalidades. Contra esta degeneración sí que hay que rebelarse. 


\section{Las necesidades básicas}

Reiteradamente hemos hablado de las necesidades básicas del hombre. En base a su «olvido» criticamos la sociedad y Estado actuales: el modelo que nos ofrece la sociedad de consumo se caracteriza por el desencanto, la tristeza, la penuria interna (y a veces externa). ¿Cuáles son esas necesidades «absolutas»? Dada la complejización de nuestra sociedad, adquiere visos dramáticos el «redescubrimiento» de esas necesidades, para que desde su conocimiento el sistema vuelva a nuestras manos. Tanto más urgente es esta tarea cuanto mayor es el número de teorías que «olvidan» el sistema hombre, que elucubran desde el vacío de una conciencia social perdida: hedonismo materialista, consumismo, etc.

En primer lugar habría que aclarar cuál es la finalidad de la sociedad y, por lo tanto, del Estado. Creo que está suficientemente demostrado que el objeto último de la sociedad es el hombre, en concreto su plena realización como ser social.

Establecido esto, tendríamos que clarificar sus necesidades básicas, y la forma de solucionarlas: tipología, diferentes concepciones antropológicas, papel de la sociedad y del Estado, etc. El tema es demasiado amplio y complejo: las necesidades básicas del hombre, y su satisfacción, son objeto de estudio tanto de la sociología como de la psicología, de la antropología, y aun de la ética. Tras la breve reflexión de estas páginas el tema queda abierto para investigaciones futuras.

\section{Hacia un nuevo método...}

La raíz del conflicto entre la realización de la persona y la sociedad reside para mí en el distanciamiento progresivo que se da en la actualidad entre el bien social objetivo y la conciencia que tienen de él los grupos sociales. Dicho de otro modo, la pérdida de la conciencia social, de la responsabilidad social, del conocimiento de la realidad social y de los medios de «reorientación» de la misma. En definitiva, del olvido de lo que es ser hombre, y de la finalidad primera de la sociedad.

Las distintas concepciones del hombre, de su realización, influyen de manera preeminente en este orden de cosas: individualismo, hedonismo «salvaje», etc. Ahora bien, todas estas teorías tienen una piedra de toque: el propio hombre, sus frustraciones en un caso, o su plenitud en otro...

El método de juicio y verificación que yo propongo se denominaría de la «evidencia social»: proceso educativo de esta evidencia, que aclarara la conciencia social «nublada» de grupos y personas sociales, piedra de toque de tanta teoría empobrecedora. 